\title{
A View Beyond HbA1c: Role of Continuous Glucose Monitoring
}

\author{
Haleh Chehregosha · Mohammad E. Khamseh · Mojtaba Malek • \\ Farhad Hosseinpanah · Faramarz Ismail-Beigi
}

Received: February 26, 2019 / Published online: April 29, 2019

(C) The Author(s) 2019

\begin{abstract}
Hemoglobin A1C (HbA1c) is used as an index of average blood glucose measurement over a period of months and is a mainstay of blood glucose monitoring. This metric is easy to measure and relatively inexpensive to obtain, and it predicts diabetes-related microvascular complications. However, HbA1c provides only an approximate measure of glucose control; it does not address short-term glycemic variability $(\mathrm{GV})$ or hypoglycemic events. Continuous
\end{abstract}

Enhanced Digital Features To view enhanced digital features for this article go to https://doi.org/10.6084/ m9.figshare.7977311.

H. Chehregosha · M. E. Khamseh ( $\varangle)$

Endocrine Research Center (Firouzgar), Institute of Endocrinology and Metabolism, Iran University of Medical Sciences (IUMS), Tehran, Iran

e-mail: mekhamseh@gmail.com;

khamseh.m@iums.ac.ir

M. Malek

Research Center for Prevention of Cardiovascular Disease, Institute of Endocrinology and Metabolism, Iran University of Medical Sciences (IUMS), Tehran, Iran

F. Hosseinpanah

Obesity Research Center, Research Institute for Endocrine Sciences, Shahid Beheshti University of Medical Sciences, Tehran, Iran

F. Ismail-Beigi

School of Medicine, Case Western Reserve

University, Cleveland, OH, USA glucose monitoring (CGM) is a tool which helps clinicians and people with diabetes to overcome the limitations of HbA1c in diabetes management. Time spent in the glycemic target range and time spent in hypoglycemia are the main CGM metrics that provide a more personalized approach to diabetes management. Moreover, the glucose management indicator (GMI), which calculates an approximate HbA1c level based on the average CGM-driven glucose level, facilitates individual decision-making when the laboratory-measured $\mathrm{HbA} 1 \mathrm{c}$ and estimated HbA1c are discordant. GV, on the other hand, is a measure of swings in blood glucose levels over hours or days and may contribute to diabetesrelated complications. In addition, addressing $\mathrm{GV}$ is a major challenge during the optimization of glycemia. The degree of GV is associated with the frequency, duration, and severity of the hypoglycemic events. Many factors affect $\mathrm{GV}$ in a patient, including lifestyle, diet, the presence of comorbidities, and diabetes therapy. Recent evidence supports the use of some glucose-lowering agents to improve GV, such as the new ultra-long acting insulin analogs, as these agents have a smoother pharmacodynamic profile and improve glycemic control with fewer fluctuations and fewer nocturnal hypoglycemic events. These newer glucoselowering agents (such as incretin hormones or sodium-glucose cotransporter 2 inhibitors) can also reduce the degree of GV. However, randomized trials are needed to evaluate the effect 
of GV on important diabetes outcomes. In this review, we discuss the role of HbA1c as a measure of glycemic control and its limitations. We also explore additional glycemic metrics, with a focus on time (duration) in glucose target range, time (duration) in hypoglycemia, GV, GMI, and their correlation with clinical outcomes.

Keywords: Continuous glucose monitoring; HbA1c; Glucose management indicator; Glycemic variability; Time in hypoglycaemia; Time in range

\section{INTRODUCTION}

Globally, the incidence and prevalence of both types 1 and 2 diabetes mellitus (DM) have risen dramatically over the past two decades and they are expected to keep rising in the future. Diabetes is associated with various chronic complications that result in increased morbidity and mortality [1, 2].

In addition to persons with diabetes receiving appropriate and timely medical management, they also require assessment of their glycemic control. The monitoring of glycemic status is considered to be a cornerstone of diabetes care. Analysis of the glucose data provides an assessment of the efficacy of therapy and guides adjustments in lifestyle and medications to achieve the best possible blood glucose control in a safe manner. Primary techniques to assess the effectiveness of glycemic control include patient self-monitoring of blood glucose (SMBG) and measurement of hemoglobin A1c (HbA1c) [3].

The results of the Diabetes Control and Complications Trial (DCCT) and other studies provide reliable evidence on the health benefits of normal or near-normal blood glucose levels, especially in patients receiving insulin treatment [4]. SMBG enables assessment of an individual's response to therapy. In a study of insulin-naive patients with suboptimal initial glycemic control, structured SMBG performed on 3 consecutive days reduced HbA1c by $0.3 \%$ compared with the control group [5]. Results from meta-analyses suggest that SMBG can reduce $\mathrm{HbA1c}$ by $0.25-0.3 \%$. However, SMBG alone does not lower blood glucose levels. To be useful, the information must be integrated into clinical and self-management programs [3]. There are additional limitations. SMBG shows a single "point-in-time" measurement and does not provide any data on the direction or rate of change of glucose levels. In addition, obtaining adequate data is dependent upon the patient's adherence to self-monitoring. Consequently, SMBG often fails to detect nocturnal and/or asymptomatic hypoglycemia [6, 7].

HbA1c represents the percentage of circulating hemoglobin that is glycated. Glycation is a non-enzymatic process and is a measure of glucose levels over time [8]. As a biomarker, it reflects the average plasma glucose over the previous 8-12 weeks [9]. It is currently used for both the diagnosis and management of diabetes [8] and is recommended as a gold standard in the assessment of diabetes-related outcomes $[9,10]$. Historically, the elevated level of HbA1c in diabetic patients was reported first by Rahbar and associates in 1968, and over the next decades it became arguably the most important indicator of blood glucose control. It is widely used to judge the adequacy of diabetes treatment [11]. Nevertheless, for a given HbA1c, there is a wide range of mean glucose concentration values, and for any given mean glucose value there is a wide range of HbA1c values [12].

The test is easy to administer, cost-effective, and relatively inexpensive [13]. Furthermore, there is a curvilinear relationship between HbA1c and microvascular complications. Three landmark trials in patients with type $2 \mathrm{DM}$ (ACCORD, ADVANCE, and VADT) showed that lower $\mathrm{HbA} 1 \mathrm{c}$ levels are associated with reduced onset or progression of some microvascular complications [14-16]. To the contrary, elevated $\mathrm{HbA1c}$ is also regarded as an independent risk factor for coronary heart disease and stroke. One of the major limitations of HbA1c is that it does not reflect glycemic excursions, i.e., intraday and inter-day glycemic changes, which have been linked to both microvascular and macrovascular complications [17]. Furthermore, it is an unreliable measure in patients with anemia, certain hemoglobinopathies, liver disease, and iron deficiency. Moreover, it does not 
provide detailed information on how to adjust the treatment regimen.

The literature suggests that ethnic and racial differences exist in the degrees of glycation that affect the accuracy of HbA1c measurements. For example, HbA1c concentration is higher (by $0.2-0.4 \%)$ in US African Americans and Hispanic populations than in Caucasians. The impact of age and race on HbA1c is currently under discussion. Some studies show that the HbA1c concentration increases by approximately $1 \mathrm{mmol} / \mathrm{mol}(0.1 \%)$ per decade of life and that this age effect results from decreasing red blood cell count with age $[18,19]$. Despite these limitations, the diabetes research community has made considerable progress in agreeing upon core glycemic outcomes beyond HbA1c to overcome the above limitations [10].

In this review, we address the latest evidence for the use of alternative metrics of glucose control in patients with diabetes. We also discuss the impact of novel metrics on diabetesrelated complications, the usefulness of CGM in the treatment of type $2 \mathrm{DM}$, and the impact of newer glucose-lowering agents on alternative glycemic metrics beyond HbA1c.

This article is based on previously conducted studies and does not contain any studies with human participants or animals performed by any of the authors.

\section{CONTINUOUS GLUCOSE MONITORING}

Continuous glucose monitoring (CGM) does not measure blood sugar concentrations, rather it measures interstitial glucose levels. CGM data for a period of 10-14 days provide a good estimate of CGM metrics for a 3-month period. HbA1c can be estimated (eA1c) if 70\% of the CGM data are available. Real-time CGM (rtCGM) data can help individuals learn how dramatically their blood glucose level can rise after certain meals or how it is affected by stress or exercise [20]. Therefore, CGM seems to be the best example of precision medicine in diabetes [12]. However, interstitial glucose readings have a time lag of approximately 15 min compared to blood glucose readings; consequently, CGM results do not always match finger stick blood glucose readings.

Different definitions are currently used for CGM systems. Intermittently viewed CGM (iCGM), known comercially as flash glucose monitoring (FGM), shows continuous glucose measurements retrospectively. This type of monitoring may be described as a separate entity from CGM, occupying a position somewhat between a traditional blood glucose meter and a CGM system. On the other hand, realtime CGM (rtCGM) provides real-time data on glucose trends, direction, and rate of change [17]. Although both systems provide the means to move beyond the HbA1c measurement as the sole marker of glycemic control, utilization of CGM as a biomarker of blood glucose levels has remained fairly limited to date [21].

Riddlesworth et al. [22], and the "Beyond A1c Writing Group" [10] recently declared that HbA1c does not accurately reflect the glucose pattern at the individual level. Furthermore, the Steering Committee of decision-making for the "Type 1 Diabetes Outcomes Program" recommended the use of defined clinically meaningful outcomes beyond HbA1c in research, development, and evaluation of therapies for type $1 \mathrm{DM}$. The novel CGM outcomes include hypoglycemia, hyperglycemia, time in glycemia range, diabetic ketoacidosis, and patient-reported outcomes [23]. A summary of the new definitions of clinically meaningful outcomes is shown in Table 1.

Apart from providing the mean glucose concentration, CGM profiles provide additional details on the patterns of glycemic excursions, as well as potentially dangerous high or low glucose concentrations that are often missed with SMBG [21, 22, 24]. Examples of some profiles with the same HbA1c are shown in Fig. 1.

\section{Advantages and Limitations of CGM}

Continuous glucose monitoring provides valuable information unattainable by SMBG, including coverage of the individual data throughout the glucose monitoring, detection of glycemic variability (GV) and the rate of change over time, as well as time spent in hypo- 
Table 1 New definitions of hypoglycemia, hyperglycemia, and time in glycemic range

\begin{tabular}{|c|c|}
\hline Outcome & Definition \\
\hline \multirow[t]{3}{*}{ Hypoglycemia } & $\begin{array}{l}\text { Level 1: glucose }<70 \mathrm{mg} / \mathrm{dL}(3.9 \mathrm{mmol} / \\
\text { L) and glucose } \geq 54 \mathrm{mg} / \mathrm{dl} \\
(3.0 \mathrm{mmol} / \mathrm{L})\end{array}$ \\
\hline & $\begin{array}{l}\text { Level 2: glucose }<54 \mathrm{mg} / \mathrm{dL}(3.0 \mathrm{mmol} / \\
\text { L) }\end{array}$ \\
\hline & $\begin{array}{l}\text { Level 3: a severe event characterized by } \\
\text { altered mental and/or physical status } \\
\text { requiring assistance }\end{array}$ \\
\hline \multirow[t]{2}{*}{ Hyperglycemia } & $\begin{array}{l}\text { Level } 1 \text { (elevated glucose): } \\
\text { glucose }>180 \mathrm{mg} / \mathrm{dL}(10 \mathrm{mmol} / \mathrm{L}) \\
\text { and glucose } \leq 250 \mathrm{mg} / \\
\text { dlL }(13.9 \mathrm{mmol} / \mathrm{L})\end{array}$ \\
\hline & $\begin{array}{l}\text { Level } 2 \text { (very elevated glucose): } \\
\text { glucose }>250 \mathrm{mg} / \mathrm{dL}(13.9 \mathrm{mmol} / \mathrm{L})\end{array}$ \\
\hline Time in range & $\begin{array}{l}\text { Percentage of readings in the range of } \\
70-180 \mathrm{mg} / \mathrm{dL}(3.9-10.0 \mathrm{mmol} / \mathrm{L}) \text { per } \\
\text { unit of time }\end{array}$ \\
\hline
\end{tabular}

and hyperglycemia. CGM can be considered for patients with severe or nocturnal hypoglycemia, especially those with hypoglycemia unawareness [21]. Also, the GOLD [25] and DIAMOND [26] trials showed that among patients with type $1 \mathrm{DM}$ treated with multiple daily insulin injections, the use of CGM resulted in better glycemic control than did conventional treatment. Moreover, several studies have shown that the use of CGM can improve the mean amplitude of glycemic excursion (MAGE) and result in better glycemic control in persons with type $2 \mathrm{DM}$ [27-30]. A recent meta-analysis of randomized controlled trials conducted on the utility of real-time and retrospective CGM in patients with type 2 DM showed that CGM in these patients could reduce HbA1c levels and time spent with hypoglycemia [31]. Finally, CGM data can be used as a valuable tool for patient education [21].

Despite these advantages, CGM has a number of limitations, namely reimbursement

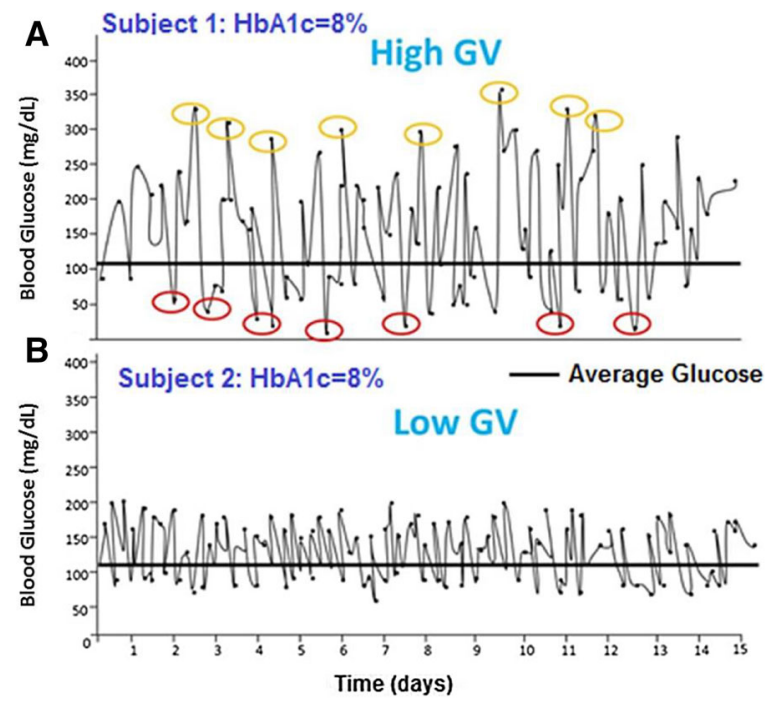

Fig. 1 Different patterns of glycemic variability $(G V)$ in two patients with same hemoglobin A1C ( $H b A l c)$. 15-day glucose traces of two patients who had identical HbAlc of $8.0 \%$ but different degrees of GV. High GV in patient 1 was reflected by numerous episodes of both hypo- and hyperglycemia (a), whereas low GV in subject 2 resulted in no such episodes (b). Patient 1 (a) had visibly higher glucose fluctuations than patient 2 (b) that resulted in seven episodes of moderate hypoglycemia $(\leq 50 \mathrm{mg} / \mathrm{dL})$ and eight episodes of moderate hyperglycemia $(\geq 350 \mathrm{mg} /$ dL)

issues, the periodic replacement of sensors for long-duration implantable CGM devices, and the need for development of clinical guidelines on the role of CGM in the management of people with type $2 \mathrm{DM}[21,32]$. However, recent advancements in technology and relevant evidence from the literature have addressed several of these issues. A new generation of insulin pumps with automated suspension of insulin infusion in response to observed or predicted hypoglycemia as well as the development of closed-loop insulin delivery systems are expected to dramatically increase the clinical utility and impact of CGM [21].

\section{TIME IN RANGE AND HYPOGLYCEMIA}

Both rtCGM and iCGM facilitate monitoring of time spent in the target blood glucose range, 
referred to as the "time in range." However, only rtCGM can warn users if the blood glucose level is trending toward hypoglycemia or hyperglycemia. Using iCGM data, the trends can only be viewed retrospectively [17]. In addition, retrospective analysis of CGM data quantifies patterns of hypo- and hyperglycemia and GV. In short, CGM helps individuals with diabetes and clinicians to personalize their management strategies [22]. The time in range target is becoming the new standard for patients and healthcare providers [23]. The use of CGM is associated with increased time in range and reduced incidence of severe hypoglycemic events [33].

\section{EA1C AND GLUCOSE MANAGEMENT INDICATOR}

\section{eA1c and Measured Laboratory HbA1c}

With the increasing use of CGM to facilitate safe and effective diabetes management, it is important to understand how CGM metrics, namely eA1c, correlates with and can be used as a metric instead of or in addition to HbA1c. eA1c for every single patient is calculated based on CGM readings from a population of individuals. In some patients, the results of a laboratory-measured $\mathrm{HbA} 1 \mathrm{c}$ and $\mathrm{eA} 1 \mathrm{c}$ are approximately the same, but in others, the eA1c may be either higher or lower than the measured HbA1c. This issue is discussed in a recent publication by Beck et al. [12] who suggested that a laboratory-measured HbA1c of $8.0 \%$ can be associated with a range of eA1c of $7-8.5 \%$. The discordance can be confusing for both patients and clinicians. For this reason, the US Food and Drug Administration determined that the nomenclature of eA1c needs to be changed, leading to the generation of a new index: the glucose management indicator (GMI). The results from the HypoDE study and from a prior study were used to generate a pooled equation to convert CGM-derived mean glucose to the GMI [22, 34]:

$$
\begin{aligned}
\mathrm{GMI}(\%)= & 3.31+0.02392 \\
& \times \text { mean glucose in } \mathrm{mg} / \mathrm{dL} .
\end{aligned}
$$

Based on this formula, an increment of $25 \mathrm{mg} / \mathrm{dL}$ in mean glucose corresponds to a $0.6 \%$ increase in GMI [22].

\section{GMI Versus Laboratory-Measured HbA1c}

The difference between the GMI and laboratorymeasured $\mathrm{HbA1c}$ in 528 individuals with diabetes who had both values measured concurrently was evaluated by Bergenstal et al. and showed in 19\% of the measurements, the GMI, calculated from CGM-derived mean glucose, and laboratory-measured HbA1c have identical values; however, in $28 \%$ of the measurements, the difference between the two values is $>0.5 \%$. Therefore, the introduction of GMI as a new glucose index could be an important step in the direction of a more personalized diabetes management program. If a person has a GMI that is always considerably lower than a measured HbA1c, the healthcare professional has to be careful not to set the therapeutic goal too low to overcome the risk of hypoglycemia. On the other hand, if the GMI is higher, it may be safe to set the HbA1c target slightly lower to minimize excessive hyperglycemia [20, 33]. It should be noted that current evidence indicates that the difference in laboratory-measured HbA1c and GMI remains relatively stable for each individual over time.

\section{GLYCEMIC VARIABILITY}

Hypoglycemia and glucose variability are considered to be the major challenges during optimization of glycemic control [35, 36]. Individualization of glycemic targets, namely HbA1c, may not always translate into improved clinical outcomes. Thus, glycemia metrics beyond HbA1c may be used to predict the risk of diabetes-related complications. In this context, CGM metrics can be associated with micro- and macrovascular complications in diabetic patients. For example, GV is associated with an increased risk of adverse cardiovascular outcomes due primarily to hypoglycemia [37]. 
Furthermore, it has been reported that patients with more advanced diabetic retinopathy spend significantly less time in the target range and have higher measures of GV [38].

A simple definition of $\mathrm{GV}$ is the degree by which a patient's blood glucose level fluctuates between high and low levels [39]. The percentage coefficient of variation $(\% \mathrm{CV})$ is defined as: $\% \mathrm{CV}=[$ (standard deviation of glucose $) /($ mean glucose) $] \times 100$. It is characterized by the amplitude, frequency, and duration of fluctuations in blood glucose $[40,41]$, with a larger magnitude of GV being associated with a higher incidence of hypoglycemia [42]. To define the threshold for GV, Monnier et al. [41] showed that a percentage coefficient of variation for glucose of $36 \%$ appears to be a suitable threshold to distinguish between stable and unstable glycemia in diabetes. It appears that beyond the $36 \%$ limit, the frequency of hypoglycemia is significantly increased, especially in insulintreated subjects [17]. CGM profile and mean glucose and glycemic variability are showed in Fig. 2.
Many factors affect GV in patients, including lifestyle [43], diet, the presence of comorbidities, diabetes treatment [44], and even insulin injection technique [45]. The best method by which to assess GV is still a matter of debate.

Types of GV in terms of duration vary from the short term to the long term. In routine clinical practice, GV is often reported according as follows. Short-term GV is defined as approximately GV of 24-72 h duration; intermediateterm GV, as GV of 3 days to 1 month duration; and long-term GV, as 1 month to years, especially referring to variations in HbA1c determined at repeated clinic visits.

GV may contribute to diabetes-related complications. Several studies have reported that long-term $\mathrm{GV}$, i.e., longitudinal variations in HbA1c, are related to micro- and macrovascular complications. However, the association of varying duration and magnitude of GV with diabetes-related outcomes is still unclear. Further clinical trials that focus on the mechanism of GV are required to evaluate whether GV can be a valuable therapeutic target in patients with type 2 DM [43].

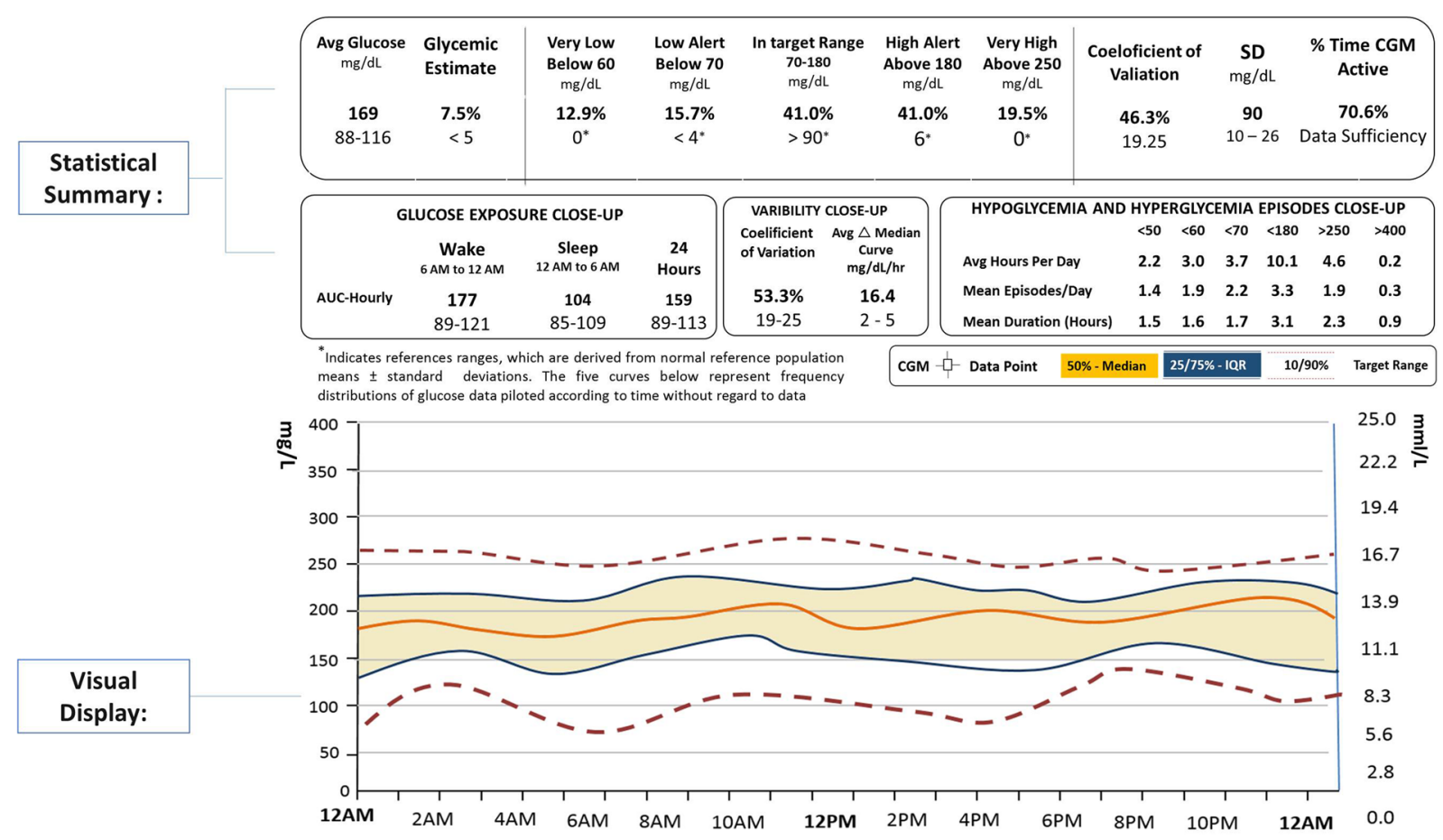

Fig. 2 The electronic CGM profile 
The Atherosclerosis Risk in Communities (ARIC) study [46] explored the association between short-term GV and diabetes-related complications by evaluating the associations of 1, 5-anhydroglucitol $(1,5-A G)$ with risk of diabetes and microvascular disease. A blood glucose level of $>180 \mathrm{mg} / \mathrm{dL}$ is associated with lower 1, 5-AG levels. The authors of this study showed that low levels of 1, 5-AG were associated with an increased risk of retinopathy and incident chronic kidney disease. Furthermore, short-term GV was associated with a range of complications, such as hypoglycemia, intensive care unit mortality, cognitive impairment, reduced quality of life, and negative mood [46-49].

In addition, intra-day and inter-day GV are significantly associated with the risk of hypoglycemia in insulin-treated patients with type 2 $\mathrm{DM}$, even after adjusting for mean blood glucose value and HbA1c. The intra-day GV prior to initiating insulin therapy is significantly associated with the risk of hypoglycemia during insulin treatment [17]. On the other hand, Feng-fei Li et al. showed that higher HbA1c values are associated with a higher 24 -h mean amplitude of glycemic excursions compared with those found at lower and moderate HbA1c values. These results led these authors to suggest that patients with higher HbA1c values should receive special therapy aimed at reducing the larger GV [50].

Large fluctuations in blood glucose are dangerous and should be considered to be an important treatment target. Achieving this treatment targets will require the use of newer glucose-lowering agents (such as incretin hormones or sodium-glucose cotransporter 2 [SGLT2]), more rapid-acting prandial insulins, stable ("flat") long-acting insulins, and more routine use of CGM in patients with type 1 and type $2 \mathrm{DM}[36]$.

Average glucose, GMI, time spent in hyperglycemia, and time spent in the target range are general indicators of overall glucose management. However, additional metrics, such as time spent in hypoglycemia and GV, supply the patient and healthcare provider with critical information beyond the $\mathrm{A} 1 \mathrm{C}$ value, by providing objective data on various safety concerns that need to be addressed in the diabetes management plan [22].

\section{Improvement of GV and Time Spent in Glycemic Target}

Recent evidence supports the use of certain glucose-lowering agents, namely, novel longacting basal insulins and dual sodium-glucose co-transporter-1 and -2 inhibitors employed in treating type $1 \mathrm{DM}$, to improve $\mathrm{GV}$ and the time spent in target range. Recently, Bergenstal et al. compared glucose control in participants with type $1 \mathrm{DM}$ receiving insulin glargine 300 units/ $\mathrm{mL}$ (Gla-300) or glargine 100 units/mL (Gla100). They showed less increase in CGM-based glucose levels in the last $4 \mathrm{~h}$ of the 24 -h injection interval, smoother average 24-h glucose profiles with less GV irrespective of injection time, and reduced nocturnal hypoglycemia with Gla-300 compared to Gla-100 [51].

According to the DEVOTE trial, insulin degludec, as an ultra-long-acting, once-daily formulation of basal insulin, was similar to insulin glargine in improving glycemic control, but with a lower risk of severe hypoglycemia [52]. Insulin degludec has also been reported to be associated with lower day-to-day variation in glucose level than insulin glargine [53]. Also, a lower incidence of global and nocturnal hypoglycemic events has been reported in patients with high GV being treated with insulin degludec compared with those receiving insulin glargine [54]. Furthermore, the authors of the VARIATION study reported the lowest GV and lowest hypoglycemia in patients with type 2 DM using the combination of basal insulin with a glucagon-like peptide-1 receptor agonist (GLP1RA) [55]. In another study, treatment with exenatide led to an improvement in glycemic excursions in patients with type 2 DM [56].

In a pooled analysis, combination treatment with sotagliflozin and insulin significantly increased the time in range and reduced postprandial glucose levels, meeting type $1 \mathrm{DM}$ glycemic control endpoints beyond a reduction in HbA1C levels [57]. Meanwhile, in type $2 \mathrm{DM}$, a randomized controlled trial comparing dapagliflozin with placebo showed an improvement 
of glycemic control and variability, using a 7-day assessment of the glycemic profile obtained from CGM [58]. In addition, dapagliflozin may reduce GV and oxidative stress in newly diagnosed type 2 DM [59].

\section{CONCLUSION}

Hemoglobin A1c is the current gold standard for assessing glycemic control. However, this index only provides an average measure of glycemic status over a period of 2-3 months. In addition, HbA1c does not address GV and hypoglycemia. In order to personalize the treatment decision, healthcare providers need to standardize the glycemic data, view the data, and use the data to achieve increased efficacy and safety for patients.

The CGM metrics provide a more personalized approach to diabetes management and resolve most of the limitations of HbA1c. CGM detects within-day and day-to-day GV, time in glycemic target, and time in hypoglycemia. These metrics may enhance a patient's selfmanagement of diabetes.

The degree of GV is potentially associated with the frequency, duration, and severity of hypoglycemic events. Moreover, GV may be linked to the pathogenesis of diabetes complications and could impact patient management and quality of life.

Newer glucose-lowering agents (SGLT inhibitors or GLP-1RAs) and novel long-acting insulin analogs with smoother pharmacodynamic profiles, and ultra-fast-acting prandial insulins could reduce the degree of GV. Randomized trials are needed to examine the relationship between new glycemic metrics and hard endpoints, such as retinopathy, nephropathy, or cardiovascular outcomes.

\section{ACKNOWLEDGEMENTS}

The authors would like to thank Dr. Zahra Emami and Ms. Leila Mahmoodi for their cooperation in manuscript preparation.
Funding. No funding or sponsorship was received for this study or publication of this article.

Authorship. All named authors meet the International Committee of Medical Journal Editors (ICMJE) criteria for authorship for this article, and take responsibility for the integrity of the work as a whole. They have given their approval for this version to be published.

Disclosures. Haleh Chehregosha, Mohammad E Khamseh, Mojtaba Malek, Farhad Hosseinpanah and Faramarz Ismail-Beigi have nothing to disclose.

Compliance with Ethics Guidelines. This article is based on previously conducted studies and does not contain any studies with human participants or animals performed by any of the authors.

Open Access. This article is distributed under the terms of the Creative Commons Attribution-NonCommercial 4.0 International License (http://creativecommons.org/licenses/ by-nc/4.0/), which permits any noncommercial use, distribution, and reproduction in any medium, provided you give appropriate credit to the original author(s) and the source, provide a link to the Creative Commons license, and indicate if changes were made.

\section{REFERENCES}

1. Zheng J, Cheng J, Wang T, Zhang Q, Xiao X. Does HbA1c level have clinical implications in diabetic patients undergoing coronary artery bypass grafting? A systematic review and meta-analysis. Int J Endocrinol. 2017;2017:1-8.

2. Adapa D, Sarangi TK. A review on diabetes mellitus: complications, management and treatment modalities. J. Med. Health. Sci. 2015;4.

3. American Diabetes Association. Standards of medical care in diabetes. Diabetes Care. 2018;41:S55-64.

4. Nathan DM, DCCT/EDIC Research Group. The diabetes control and complications trial/epidemiology of diabetes interventions and complications 
study at 30 years: overview. Diabetes Care. 2013;37:9-16.

5. Polonsky WH, Fisher L, Schikman CH, et al. Structured self-monitoring of blood glucose significantly reduces $\mathrm{A} 1 \mathrm{C}$ levels in poorly controlled noninsulintreated type 2 diabetes: results from the Structured Testing Program study. Diabetes Care. 2011;34:262-7.

6. Boland E, Monsod T, Delucia M, Brandt CA, Fernando S, Tamborlane WV. Limitations of conventional methods of self-monitoring of blood glucose: lessons learned from 3 days of continuous glucose sensing in pediatric patients with type 1 diabetes. Diabetes Care. 2001;24:1858-62.

7. Goldstein DE, Little RR, Lorenz RA, et al. Tests of glycemia in diabetes. Diabetes Care. 2004;27:1761-73.

8. Wright JJ, Hu JR, Shajani-Yi Z, Bao S. Use of continuous glucose monitoring leads to diagnosis of hemoglobin $\mathrm{C}$ trait in a patient with discrepant haemoglobin A1c and self-monitoring blood glucose. AACE Clin Case Rep. 2019;5:e31-4.

9. Sherwani SI, Khan HA, Ekhzaimy A, Masood A, Sakharkar MK. Significance of HbA1c test in diagnosis and prognosis of diabetic patients. Biomark Insights. 2016;1:95-104.

10. Beyond A1C Writing Group. Need for regulatory change to incorporate beyond A1C glycemic metrics. Diabetes Care. 2018;41:e92-94.

11. Gebel E. The start of something good: the discovery of HbA1c and the American Diabetes Association samuel rahbar outstanding discovery award. Diabetes Care. 2012;35:2429-31.

12. Beck RW, Connor CG, Mullen DM, Wesley DM, Bergenstal RM. The fallacy of average: how using HbA1c alone to assess glycemic control can be misleading. Diabetes Care. 2017;40:994-9.

13. Gupta S, Jain U, Chauhan N. Laboratory diagnosis of HbA1c: a review. J Nanomed Res. 2017;5:00120.

14. Action to Control Cardiovascular Risk in Diabetes Follow-On (ACCORDION) Eye Study Group, Action to Control Cardiovascular Risk in Diabetes FollowOn (ACCORDION) Study Group. Persistent effects of intensive glycemic control on retinopathy in type 2 diabetes in the action to control cardiovascular risk in diabetes (ACCORD) follow-on study. Diabetes Care. 2016;39:1089-100.

15. Heller SR, ADVANCE Collaborative Group. A summary of the ADVANCE trial. Diabetes Care. 2009;32:S357-61.
16. Agrawal L, Azad N, Bahn GD, et al. Long-term follow-up of intensive glycaemic control on renal outcomes in the Veterans Affairs Diabetes Trial (VADT). Diabetologia. 2018;61:295-9.

17. Danne T, Nimri R, Battelino $\mathrm{T}$, et al. International consensus on use of continuous glucose monitoring. Diabetes Care. 2017;40:1631-40.

18. Wu L, Lin H, Gao J, et al. Effect of age on the diagnostic efficiency of HbA1c for diabetes in a Chinese middle-aged and elderly population: the Shanghai Changfeng Study. PLoS One. 2017;12:e0184607.

19. Weykamp C. HbA1c: a review of analytical and clinical aspects. Ann Lab Med. 2013;33:393-400.

20. Bergenstal RM. Glycemic variability and diabetes complications: does it matter? Simply put, there are better glycemic markers! Diabetes Care. 2015;38:1615-21.

21. Rodbard D. Continuous glucose monitoring: a review of successes, challenges, and opportunities. Diabetes Technol Ther. 2016;18:S3-13.

22. Riddlesworth TD, Beck RW, Gal RL, et al. Optimal sampling duration for continuous glucose monitoring to determine long-term glycemic control. Diabetes Technol Ther. 2018;20:314-6.

23. Agiostratidou G, Anhalt H, Ball D, et al. Standardizing clinically meaningful outcome measures beyond $\mathrm{HbA} 1 \mathrm{c}$ for type 1 diabetes: A consensus report of the American Association of Clinical Endocrinologists, the American Association of Diabetes Educators, the American Diabetes Association, the Endocrine Society, JDRF International, The Leona M. and Harry B. Helmsley Charitable Trust, the Pediatric Endocrine Society, and the T1D exchange. Diabetes Care. 2017;40:1622-30.

24. Kovatchev B, Cobelli C. Glucose variability: timing, risk analysis, and relationship to hypoglycemia in diabetes. Diabetes Care. 2016;39:502-10.

25. Lind M, Polonsky W, Hirsch IB, et al. Continuous glucose monitoring vs conventional therapy for glycemic control in adults with type 1 diabetes treated with multiple daily insulin injections: the GOLD randomized clinical trial. JAMA. 2017;317:379-87.

26. Beck RW, Riddlesworth T, Ruedy K, et al. Effect of continuous glucose monitoring on glycemic control in adults with type 1 diabetes using insulin injections: the DIAMOND randomized clinical trial. JAMA. 2017;317:371-8.

27. Li FF, Fu LY, Zhang WL, et al. Blood glucose fluctuations in type 2 diabetes patients treated with multiple daily injections. J Diabetes Res. 2016;2016. 
28. Li FF, Liu BL, Zhu HH, et al. Continuous glucose monitoring in newly diagnosed type 2 diabetes patients reveals a potential risk of hypoglycemia in older men. J Diabetes Res. 2017;2740372. https:// doi.org/10.1155/2017/2740372.

29. Li FF, Jiang LL, Yan RN, et al. Effects of saxagliptin add-on therapy to insulin on blood glycemic fluctuations in patients with type 2 diabetes: A randomized, control, open-labeled trial. Medicine (Baltimore). 2016;95(43):e5229.

30. Li FF, Liu BL, Yin GP, et al. Young onset type 2 diabetic patients might be more sensitive to metformin compared to late onset type 2 diabetic patients. Sci Rep. 2017;7:16382.

31. Ida S, Kaneko R, Murata K. Utility of real-time and retrospective continuous glucose monitoring in patients with type 2 diabetes mellitus: a metaanalysis of randomized controlled trials. J Diabetes Res. 2019;2019.

32. American Diabetes Association. Standards of medical care in diabetes. Diabetes Care. 2018;41:S55-64.

33. Bergenstal RM, Beck RW, Close KL, et al. Glucose management indicator (GMI): a new term for estimating A1C from continuous glucose monitoring. Diabetes Care. 2018;41:2275-80.

34. Van Beers CA, DeVries JH, Kleijer SJ, et al. Continuous glucose monitoring for patients with type 1 diabetes and impaired awareness of hypoglycaemia (IN CONTROL): a randomised, open-label, crossover trial. Lancet Diabetes Endocrinol. 2016;4:893-902.

35. Heinemann L, Freckmann G, Ehrmann D, et al. Real-time continuous glucose monitoring in adults with type 1 diabetes and impaired hypoglycaemia awareness or severe hypoglycaemia treated with multiple daily insulin injections (HypoDE): a multicentre, randomised controlled trial. Lancet. 2018;391:1367-77.

36. Kovatchev BP. Metrics for glycaemic control-from HbA 1c to continuous glucose monitoring. Nat Rev Endocrinol. 2017;13:425-36.

37. Monnier L, Colette C, Owens DR. The application of simple metrics in the assessment of glycaemic variability. Diabetes Metab. 2018;44(4):313-9.

38. Lu J, Ma X, Zhou J, et al. Association of time in range, as assessed by continuous glucose monitoring, with diabetic retinopathy in type 2 diabetes. Diabetes Care. 2018;41:2370-6.

39. Hirsch IB. Glycemic variability and diabetes complications: does it matter? Of course it does! Diabetes Care. 2015;38:1610-4.
40. Qu Y, Jacober SJ, Zhang Q, Wolka LL, DeVries JH. Rate of hypoglycemia in insulin-treated patients with type 2 diabetes can be predicted from glycemic variability data. Diabetes Technol Ther. 2012;14:1008-12.

41. Monnier L, Colette C, Wojtusciszyn A, et al. Toward defining the threshold between low and high glucose variability in diabetes. Diabetes Care. 2017;40:832-8.

42. Kovatchev BP, Flacke F, Sieber J, Breton MD. Accuracy and robustness of dynamical tracking of average glycemia (A1c) to provide real-time estimation of hemoglobin A1c using routine self-monitored blood glucose data. Diabetes Technol Ther. 2014;16:303-9.

43. Mori H, Okada Y, Kurozumi A, Narisawa M, Tanaka Y. Factors influencing inter-day glycemic variability in diabetic outpatients receiving insulin therapy. J Diabetes Investig. 2017;8:69-74.

44. Sakamoto M. Type 2 diabetes and glycemic variability: various parameters in clinical practice. J Clin Med Res. 2018;10:737-42.

45. Yuan L, Li F, Jing $\mathrm{T}$, et al. Insulin injection technique is associated with glycemic variability in patients with type 2 diabetes. Diabetes Ther. 2018;9:2347-56.

46. Selvin E, Rawlings AM, Grams M, Klein R, Steffes M, Coresh J. Association of 1, 5-anhydroglucitol with diabetes and microvascular conditions. Clin Chem. 2014;60:1409-18.

47. Hermanides J, Vriesendorp TM, Bosman RJ, Zandstra DF, Hoekstra JB, Devries JH. Glucose variability is associated with intensive care unit mortality. Crit Care Med. 2010;38:838-42.

48. Lim JS, Kim C, Oh MS, et al. Effects of glycemic variability and hyperglycemia in acute ischemic stroke on post-stroke cognitive impairments. J Diabetes Complicat. 2018;32:682-7.

49. Inzucchi SE, Bergenstal RM, Buse JB, et al. Management of hyperglycemia in type 2 diabetes, 2015: a patient-centered approach: update to a position statement of the American Diabetes Association and the European Association for the Study of Diabetes. Diabetes Care. 2015;38:140-9.

50. Li FF, Liu BL, Yan RN, et al. Features of glycemic variations in drug naïve type 2 diabetic patients with different HbA 1c values. Sci Rep. 2017;7:1583.

51. Bergenstal RM, Bailey TS, Rodbard D, et al. Comparison of insulin glargine 300 Units $/ \mathrm{mL}$ and 100 Units/mL in adults with type 1 diabetes: continuous glucose monitoring profiles and variability using 
morning or evening injections. Diabetes Care. 2017;40:554-60.

52. Marso SP, McGuire DK, Zinman B, et al. Design of DEVOTE (trial comparing cardiovascular safety of insulin degludec vs insulin glargine in patients with type 2 diabetes at high risk of cardiovascular events)-DEVOTE 1. Am Heart J. 2016;179:175-83.

53. Iga $\mathrm{R}$, Uchino $\mathrm{H}$, Kanazawa $\mathrm{K}$, et al. Glycemic variability in type 1 diabetes compared with degludec and glargine on the morning injection: an openlabel randomized controlled trial. Diabetes Ther. 2017;8:783-92.

54. Henao-Carrillo DC, Muñoz OM, Gómez AM, et al. Reduction of glycemic variability with Degludec insulin in patients with unstable diabetes. J Clin Transl Endocrinol. 2018;12:8-12.

55. Bajaj HS, Venn K, Ye C, et al. Lowest glucose variability and hypoglycemia are observed with the combination of a GLP-1 receptor agonist and basal insulin (VARIATION Study). Diabetes Care. 2017;40:194-200.
56. Li FF, Jiang L, Fu L, et al. Exenatide add-on to continuous subcutaneous insulin infusion therapy reduces bolus insulin doses in patients with type 2 diabetes: a randomized, controlled, open-label trial. Diabetes Ther. 2017;8:177-87.

57. Danne T, Cariou B, Buse JB, et al. Increased time-inrange with sotagliflozin as adjunct therapy to insulin in adults with type 1 diabetes as demonstrated by 24 -week continuous glucose monitoring (inTandem1, inTandem2). Diabetes. 2018;67(Supplement 1). https://doi.org/10.2337/db18-1179-P.

58. Henry RR, Strange P, Zhou R, Pettus J, Shi L, Zhuplatov SB, Mansfield T, Klein D, Katz A. Effects of dapagliflozin on 24-hour glycemic control in patients with type 2 diabetes: a randomized controlled trial. Diabetes Technol Ther. 2018;20:715-24.

59. Li FF, Gao G, Li Q, et al. Influence of dapagliflozin on glycemic variations in patients with newly diagnosed type 2 diabetes mellitus. J Diabetes Res. 2016;2016:5347262. 